\title{
Preconditioned HLLE Method for Flows at All Mach Numbers
}

\author{
Soo Hyung Park* \\ Konkuk University, Seoul 143-701, Republic of Korea \\ and \\ Jae Eun Lee ${ }^{\dagger}$ and Jang Hyuk Kwon \\ Korea Advanced Institute of Science Technology, Daejeon 305-701, Republic of Korea
}

DOI: $10.2514 / 1.12176$

\begin{abstract}
A two-dimensional preconditioned multigrid Navier-Stokes solver has been developed for all Mach number viscous flows. For the computation of all Mach number flows, a local preconditioning technique with a modified reference velocity was used to extend the Harten-Lax-van Leer-Einfeldt (HLLE+) scheme, which has good characteristics for compressible viscous flows. The developed methods used to compute several representative steady problems. Grid refinement and parametric studies are performed to verify the performance. The results show that the preconditioned HLLE + scheme can be successfully applied to a wide variety of flows and that the implicit multigrid solver provides good convergence for the test problems.
\end{abstract}

\section{Introduction}

A number of numerical flux functions for inviscid fluxes have been devised as approximate solutions to Riemann problems. Harten et al.[1] suggested a mathematical theory for the upstream difference scheme and the Godunov-type scheme, denoted by the Harten-Laxvan Leer (HLL) which approximates the solution of the Riemann problem with two signal waves. A typical example of the HLL solver is the HLLE scheme [2] that proposed the bounds of signal waves by using the eigenvalues of the Roe matrix to satisfy the entropy and positivity conditions. Despite its desirable properties, it is difficult to simulate practical problems because of its highly dissipative behavior. The HLLE-modified (HLLEM) scheme [3] enhances the resolution to a level comparable to that of the Roe scheme [4]. However, several problems have been reported for this scheme [5,6]. The HLLE+ scheme [6], which successfully eliminates the erroneous dissipation and the instability of the HLLEM scheme, has been devised to predict the supersonic viscous flows accurately.

Generally, the Godunov-type schemes are unsuitable for solving low Mach number or incompressible flow problems because of the large condition number of the eigensystem of the governing equations. Time-derivative preconditioning techniques have been proposed to reduce the stiffness and convergence problems that occur at low Mach number flows. Since Viviand [7] proposed a generalized preconditioning procedure for a class of hyperbolic systems, many researchers[8-12] have conducted a number of preconditioning studies. By altering the acoustic wave speeds, their methods make the condition number bounded independently of the Mach number of the flows.

Our objectives are to modify the HLLE+ scheme [6], which is suitable for simulating supersonic viscous flows, and to assess the performance of the modified HLLE+ scheme at low Mach numbers.

Presented as Paper 2709 at the 34th AIAA Fluid Dynamics Conference and Exhibit, Portland, OR, 28 June-1 July 2004; received 12 July 2004; revision received 4 April 2006; accepted for publication 4 July 2006. Copyright (C) 2006 by the authors. Published by the American Institute of Aeronautics and Astronautics, Inc., with permission. Copies of this paper may be made for personal or internal use, on condition that the copier pay the $\$ 10.00$ per-copy fee to the Copyright Clearance Center, Inc., 222 Rosewood Drive, Danvers, MA 01923; include the code $\$ 10.00$ in correspondence with the CCC.

*Research Professor, NITRI, 1 Hwayang-dong, Gwangjin-Gu; pish@ konkuk.ac.kr. Member AIAA.

Doctoral Candidate, Department of Aerospace Engineering, 373-1 Guseong-dong, Yuseong-gu; LeeJaeeun@kaist.ac.kr.

Professor, Department of Aerospace Engineering, 373-1 Guseong-dong, Yuseong-gu; jhkwon@kaist.ac.kr. Senior Member AIAA.
Previously, Luo et al. [13] modified the signal velocities of the HLL with contact restoration (HLLC) scheme based on the eigenvalues of the preconditioned system and implemented at transonic and low speed viscous flows. As with earlier attempts [13-15] to modify shock-capturing schemes, we used the time-derivative preconditioning technique of Weiss and Smith [10] and slightly modified the reference velocity to improve its robustness both at supersonic and subsonic regions. We used the preconditioned HLLE+ scheme to compute several representative problems of low speed viscous flows. Grid refinement and parametric studies verified the results.

\section{Time-Derivative Preconditioning}

\section{Numerical Methods}

We consider the preconditioned form of the two-dimensional compressible Navier-Stokes equations,

$$
\Gamma \frac{\partial \boldsymbol{Q}}{\partial t}+\frac{\partial \boldsymbol{F}}{\partial x}+\frac{\partial \boldsymbol{G}}{\partial y}=\frac{\partial \boldsymbol{F}_{\boldsymbol{v}}}{\partial x}+\frac{\partial \boldsymbol{G}_{\boldsymbol{v}}}{\partial y}+\boldsymbol{g}
$$

where $\boldsymbol{Q}$ is the primitive flow variable vector, $\boldsymbol{F}$ and $\boldsymbol{G}$ are the inviscid fluxes in $x$ - and $y$-directions, respectively, and $\boldsymbol{F}_{\boldsymbol{v}}$ and $\boldsymbol{G}_{\boldsymbol{v}}$ are the viscous fluxes:

$$
\begin{gathered}
\boldsymbol{Q}=\left[\begin{array}{c}
p \\
u \\
v \\
T
\end{array}\right], \quad \boldsymbol{F}=\left[\begin{array}{c}
\rho u \\
\rho u^{2}+p \\
\rho u v \\
\rho u H
\end{array}\right], \quad \boldsymbol{G}=\left[\begin{array}{c}
\rho v \\
\rho u v \\
\rho v^{2}+p \\
\rho v H
\end{array}\right] \\
\boldsymbol{F}_{v}=\left[0, \sigma_{x x}, \sigma_{x y}, u \sigma_{x x}+v \sigma_{x y}-q_{x}\right]^{T} \\
\boldsymbol{G}_{v}=\left[0, \sigma_{y x}, \sigma_{y y}, u \sigma_{y x}+v \sigma_{y y}-q_{y}\right]^{T}
\end{gathered}
$$

Here $\rho, p$, and $T$ are the density, pressure, and temperature, and $u$ and $v$ are the Cartesian velocity components. $H=e+p / \rho$ is the total enthalpy and $e$ is the total energy. The quantities $\sigma_{i j}$ and $q_{i}$ are the viscous stresses and heat fluxes in each direction. The laminar viscosity coefficient is evaluated by using the Sutherland's law. The Prandtl number based on reference transport properties is 0.7. $g$ denotes the source vector, such as a gravitational body force. Specially, pressure and temperature terms in the primitive variable set are expressed as a perturbed form to decrease roundoff errors at subsonic flows $[9,16]$.

The preconditioning matrix $\Gamma$ is used to scale acoustic wave speeds by introducing a preconditioned velocity scale. The matrix 
chosen is originally proposed by Choi and Merkle [9] and extended by Weiss and Smith [10]. $\Gamma$ is given by

$$
\left[\begin{array}{cccc}
\Theta+1 /(R T) & 0 & 0 & -\rho / T \\
u[\Theta+1 /(R T)] & \rho & 0 & -\rho u / T \\
v[\Theta+1 /(R T)] & 0 & \rho & -\rho v / T \\
H[\Theta+1 /(R T)]-1 & \rho u & \rho v & \rho\left[C_{p}-H / T\right]
\end{array}\right]
$$

where the parameter $\Theta$ is defined as

$$
\Theta=\frac{1}{U_{r}^{2}}-\frac{1}{a^{2}}
$$

with $a$ is the speed of sound.

The reference velocity, $U_{r}$, has been chosen basically to avoid unstable behaviors at near-stagnation regions and to recover the original governing equations at supersonic flow regions $[11,17]$. The reference velocity should not drop below the local convection or diffusion velocity. For low Reynolds number viscous flows, the reference velocity should not be smaller than the local diffusion velocity, $\mu / \rho \Delta d$, where $\Delta d$ is a intercell length scale. An additional limitation on $U_{r}$ has been imposed to increase the numerical stability. The limitation prohibits amplification of the pressure perturbations in the stagnation regions [12]. Therefore, the restriction on $U_{r}$ becomes

$$
U_{r}=\min \left[a, \max \left(|\boldsymbol{U}|, K U_{\infty}, \frac{\mu}{\rho \Delta d}, \epsilon \sqrt{\frac{|\Delta p|}{\rho}}\right)\right]
$$

where $K$ is arbitrary but fixed at 0.5 for the global cutoff, and $|\Delta p|$ is obtained as the maximum for all the faces of a given cell [17]. As underlined in [17], this formulation with the pressure gradient sensor can lead to unstable behavior, particularly in the presence of strong acoustic waves. The scaling parameter $\epsilon$, which typically ranges from 0.001 to 0.1 , should be carefully tuned to prevent the instability because of its sensitivity, especially when a multigrid algorithm is applied. This instability can be easily prevented by exploiting the pressure perturbation itself rather than the neighboring pressure gradients, which are expressed as follows:

$$
U_{r}=\min \left[a, \max \left(|\boldsymbol{U}|, K \boldsymbol{U}_{\text {cut }}, \frac{\mu}{\rho \Delta d}, \sqrt{\frac{\left|p^{\prime}\right|}{\rho}}\right)\right]
$$

where $\boldsymbol{U}_{\text {cut }}=\min \left(U_{\infty}, a_{\infty}\right)$ and $p^{\prime}=\left(p_{l}+p_{r}\right) / 2 . p_{l}$ and $p_{r}$ have the meaning of the left and right gauge pressures at the adjacent cells, respectively. The optimized definition of the reference velocity, Eq. (8), can improve the robustness of an implicit multigrid algorithm without the sensitive scaling parameter $\epsilon$. Moreover, the modified parameter $K \boldsymbol{U}_{\text {cut }}$ becomes small enough to activate preconditioning in the subsonic regions dominantly supersonic flows such as blunt-body flows by employing freestream speed of sound.

\section{Godunov-Type Flux-Splitting Schemes}

Numerical flux functions have been constructed for onedimensional Euler equations because the viscous fluxes can be discretized by central differencing. Equation (1) can be integrated cellwise in one-dimensional space domain, then the discretized governing equation yields in a conservative form:

$$
\boldsymbol{W}_{i}^{n+1}=\boldsymbol{W}_{i}^{n}-\frac{\Delta t}{\Delta x}\left(\boldsymbol{F}_{i+1 / 2}^{n}-\boldsymbol{F}_{i-1 / 2}^{n}\right)
$$

where $\boldsymbol{W}$ is the conservative flow variable vector,

$$
\boldsymbol{W}=\left[\begin{array}{llll}
\rho & \rho u & \rho v & \rho e
\end{array}\right]^{T}
$$

The HLL Riemann solver [1] approximates the solution of the Riemann problem with two waves propagating at speeds of $b^{r}$ and $b^{l}$, as shown in Fig. 1. They are the lower and upper bounds for the physical signal speeds with which the information of the initial discontinuity is transported. To satisfy the entropy and the positivity conditions, Einfeldt [2] suggested adequate bounds by making use of

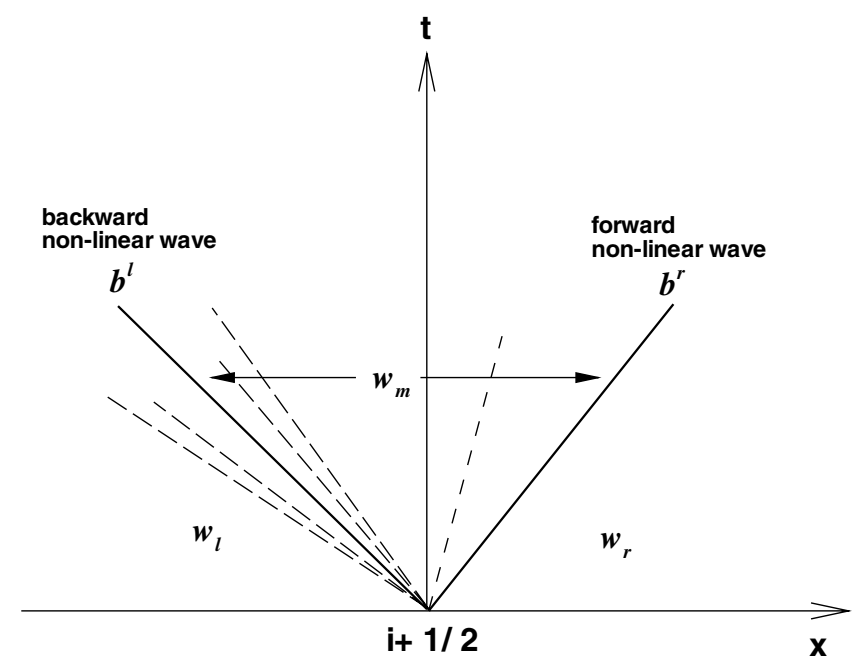

Fig. 1 The approximate solution of HLL Riemann solver.

the Roe-averaged eigenvalues, $\hat{\lambda}_{p}=\{\hat{u}, \hat{u}+\hat{a}, \hat{u}-\hat{a}, \hat{u}\}$, where $\hat{a}$ is the speed of sound at the cell interface and the subscript $p$ varies from 1 to 4 . The superscript ${ }^{\wedge}$ denotes the Roe-averaged values throughout this paper. The HLLE scheme does not violate the positivity and the entropy conditions nor suffer instability at strong shocks. However, the contact discontinuities are smeared excessively because it is very dissipative regardless of the chosen bounds. The HLLEM scheme [3] improves the resolution of contact discontinuity by reusing the information of contact discontinuity.

The schemes described in the preceding section can be unified and characterized by using control parameters. This enables us to analyze the dissipation mechanisms of each scheme. The numerical flux of the unified Godunov-type schemes is defined by

$$
\begin{aligned}
& \boldsymbol{F}_{i+1 / 2}=\frac{b^{+} \boldsymbol{F}\left(\boldsymbol{W}_{l}\right)-b^{-} \boldsymbol{F}\left(\boldsymbol{W}_{r}\right)}{b^{+}-b^{-}}+\frac{b^{+} b^{-}}{b^{+}-b^{-}}\left\{\left(\boldsymbol{W}_{r}-\boldsymbol{W}_{l}\right)\right. \\
& \left.-\sum_{p=1,4} \bar{\delta} \alpha^{p} \boldsymbol{T}^{p}\right\}
\end{aligned}
$$

with $b^{+}=\max \left\{b^{r}, 0.0\right\}$ and $b^{-}=\min \left\{b^{l}, 0.0\right\}$, where $b^{r}$ and $b^{l}$ are defined to be

$$
b^{r}=\max \left\{\hat{\lambda}_{2}, \mathbf{C}^{+}\right\}, \quad b^{l}=\min \left\{\hat{\lambda}_{3}, \mathbf{C}^{-}\right\}
$$

Here $\boldsymbol{T}^{p}(p=1,2,3,4)$ are the right eigenvectors of the flux Jacobian evaluated at intermediate states. $\alpha^{p}$ are coefficients of the projection of $\boldsymbol{W}_{r}-\boldsymbol{W}_{l}$ onto $\boldsymbol{T}^{p}$ :

$$
\boldsymbol{W}_{r}-\boldsymbol{W}_{l}=\sum_{p=1}^{4} \alpha^{p} \boldsymbol{T}^{p}
$$

Because $b^{+}$and $b^{-}$are positive and negative quantities, respectively, the last term in Eq. (11) is of an antidiffusive nature. The antidiffusion coefficient $\bar{\delta}$ is defined such that they can take out excess dissipation in linear degenerated fields:

$$
\bar{\delta}=\frac{\hat{a}}{|\bar{u}|+\hat{a}}
$$

where the speed $\bar{u}$ is defined as the approximate speed of the contact discontinuity.

According to the definition of $\boldsymbol{C}^{+}, \boldsymbol{C}^{-}$and $\bar{u}$, different dissipation mechanisms can be described as follows:

HLLE:

$$
C^{+}=u_{r}+a_{r}, \quad C^{-}=u_{l}-a_{l}, \quad|\bar{u}|=\infty \quad \text { so that } \bar{\delta}=0.0
$$




\section{HLLEM:}

$$
C^{+}=u_{r}+a_{r}, \quad C^{-}=u_{l}-a_{l}, \quad|\bar{u}|=\left|\frac{b^{r}+b^{l}}{2}\right|
$$

Roe:

$$
C^{+}=\hat{\lambda}_{2}, \quad C^{-}=\hat{\lambda}_{3}, \quad|\bar{u}|=|\hat{u}|
$$

It is noted that the numerical dissipation mechanism can be understood mainly through the antidiffusion terms which are explicitly identified. This is important in constructing a new HLL solver which captures strong shocks without encountering shock instability nor contaminating the inherent resolution of the scheme in viscous flows. We have proposed an accurate HLLE method which enhances the dissipation mechanism of antidiffusion terms, especially for viscous flows. More detailed description can be found in [6]. For brevity, it is denoted by HLLE+ in this paper. HLLE + scheme without any switching mechanism is described by the following parameters:

$$
C^{+}=u_{r}+a_{r}, \quad C^{-}=u_{l}-a_{l}, \quad|\bar{u}|=|\hat{u}|
$$

The original version of the HLLE+ scheme has a switching mechanism based on a pressure sensor. It has been found in our numerical experiments that the switching mechanism is no longer needed in the framework of a properly preconditioned system.

\section{Preconditioned HLLE+ Formulation}

Time-derivative preconditioning changes the eigensystem of the governing equations so that the eigenvalues of the system have the same order as the local convective velocity. Shock-capturing methods should therefore be modified with respect to the preconditioned system. The eigenvalues of the preconditioned system are $\Lambda_{p}^{\prime}\left(\Gamma^{-1} \partial F / \partial Q\right)=\left\{u, u^{\prime}+a^{\prime}, u^{\prime}-a^{\prime}, u\right\}$, where

$$
\begin{gathered}
u^{\prime}=u(1-\alpha), \quad a^{\prime}=\sqrt{\alpha^{2} u^{2}+U_{r}^{2}} \\
\alpha=\frac{1-\left(U_{r} / a\right)^{2}}{2}
\end{gathered}
$$

The HLLE + scheme can be modified to perform well at very low Mach numbers by replacing the signal velocities with the preconditioned signal velocities and by replacing the eigenvector matrices with the preconditioned matrices. The resulting HLLE+ scheme can then be expressed as

$$
\begin{aligned}
& \boldsymbol{F}_{i+1 / 2}=\frac{b^{+} \boldsymbol{F}\left(\boldsymbol{W}_{l}\right)-b^{-} \boldsymbol{F}\left(\boldsymbol{W}_{r}\right)}{b^{+}-b^{-}}+\frac{b^{+} b^{-}}{b^{+}-b^{-}}\left\{\left(\boldsymbol{W}_{r}-\boldsymbol{W}_{l}\right)\right. \\
& \left.-\sum_{p=1,4} \bar{\delta} \alpha_{\Gamma}^{p} \Gamma \boldsymbol{T}_{\Gamma}^{p}\right\}
\end{aligned}
$$

where

$$
\boldsymbol{W}_{r}-\boldsymbol{W}_{l}=\sum_{p=1}^{4} \alpha_{\Gamma}^{p} \Gamma \boldsymbol{T}_{\Gamma}^{p}
$$

and the preconditioned signal velocities $b^{r}$ and $b^{l}$ :

$$
\begin{gathered}
b^{r}=\max \left\{\hat{u}^{\prime}+\hat{a}^{\prime}, u_{r}^{\prime}+a_{r}^{\prime}\right\} \\
b^{l}=\min \left\{\hat{u}^{\prime}-\hat{a}^{\prime}, u_{l}^{\prime}-a_{l}^{\prime}\right\} \\
\bar{\delta}=\frac{\hat{a}^{\prime}}{\left|\bar{u}^{\prime}\right|+\hat{a}^{\prime}}
\end{gathered}
$$

The accuracy of the Godunov-type scheme is increased to the second-order by using the third-order MUSCL with the continuous
van-Albada limiter [18]. The simple central differencing is applied to obtain the variable gradients of the viscous fluxes.

It is noted that $\alpha_{\Gamma}^{p}$ and $\Gamma \boldsymbol{T}_{\Gamma}^{p}$ in the Eq. (22) can be compared with $\alpha^{p}$ and $\boldsymbol{T}^{p}$ in the Eq. (13) as shown in the Appendix.

\section{Time-Stepping Schemes}

In this paper, a multigrid diagonalized alternate directional implicit (DADI) method $[19,20]$ is applied to find steady-state solutions. The following integral form of the governing equations is considered over a control volume $\mathcal{V}(t)$ :

$$
\Gamma \frac{\mathrm{d}}{\mathrm{d} t} \int_{\mathcal{V}(t)} \boldsymbol{Q} d \mathcal{V}+\int_{\partial \mathcal{V}(t)}\left[\boldsymbol{F}-\boldsymbol{F}_{\boldsymbol{v}}\right] d \mathcal{S}=0
$$

An implicit scheme can be written as

$$
\left[\boldsymbol{I}+\Delta t \frac{\partial \boldsymbol{R}}{\partial \boldsymbol{Q}}\right] \Delta \boldsymbol{Q}=-\Delta t \Gamma^{-1} \boldsymbol{R}\left(\boldsymbol{q}^{m}\right)
$$

By using ADI method, Eq. (27) can be factorized as follows:

$$
\left\{\boldsymbol{I}+\Delta t \boldsymbol{A}_{\Gamma \xi}\right\}\left\{\boldsymbol{I}+\Delta t \boldsymbol{A}_{\Gamma \eta}\right\} \Delta \boldsymbol{Q}=-\Delta t \Gamma^{-1} \boldsymbol{R}
$$

Here, $\boldsymbol{A}_{\Gamma}=\Gamma^{-1}(\partial \boldsymbol{R} / \partial \boldsymbol{Q})$ are the Jacobian matrix of the residual vector in each direction. Finally, the preconditioned implicit formulation, (28), is expressed in a diagonalized ADI form by using the similarity transformation [21,22]:

$$
\begin{aligned}
& \boldsymbol{T}_{\Gamma \xi}\left[\boldsymbol{I}+\Delta t\left\{\nabla^{-} \Lambda_{\xi}^{\prime+}+\nabla^{+} \Lambda_{\xi}^{\prime-}-\bar{\delta}_{\xi}^{2} r\left(A_{v}\right)\right\}\right] \boldsymbol{T}_{\Gamma \xi}^{-1} \cdot \boldsymbol{T}_{\Gamma \eta}[\boldsymbol{I} \\
& \left.\quad+\Delta t\left\{\nabla^{-} \Lambda_{\eta}^{\prime+}+\nabla^{+} \Lambda_{\eta}^{\prime-}-\bar{\delta}_{\eta}^{2} r\left(A_{v}\right)\right\}\right] \boldsymbol{T}_{\Gamma \eta}^{-1} \cdot \Delta \boldsymbol{Q}=-\Delta t \Gamma^{-1} \boldsymbol{R}
\end{aligned}
$$

where $\nabla^{ \pm}$mean the backward and forward difference operators and $\Lambda_{i}^{\prime \pm}$ are the preconditioned eigenvalues in each direction. $\bar{\delta}_{i}^{2} r\left(A_{v}\right)$ operators express the central differencing of viscous fluxes. More detailed description can be found in [22]. Unlike inviscid terms, the contribution of viscous terms is not simultaneously diagonalizable, and it must be added to the implicit part only by an approximation of spectral radius scaling:

$$
r\left(A_{v}\right)=\left(\frac{\mu}{P r}\right) \frac{\gamma}{\rho}
$$

For a similar reason, the local time-stepping is applied to $\Delta t$, which is defined by the CFL number over the summation of the spectral radii of the inviscid and viscous flux vectors:

$$
\Delta t=\frac{\mathrm{CFL}}{\sum_{i}\left[\left(\left|u_{i}^{\prime}\right|+a^{\prime}\right)+r\left(A_{v}\right)\right]}
$$

A full approximate storage (FAS) multigrid algorithm [20] is used for the steady-state computation. Because the present V-cycle multigrid algorithm concentrates on the acceleration of wave propagation rather than high frequency damping, different numbers of time-stepping along with different CFL numbers are applied according to the grid level where $L$ is the grid level and $L=1$ is the finest level. In this work, two time-steps are advanced in the coarse levels. In addition, the CFL number can be increased according to the grid level. When the CFL number of the finest level is $\mathrm{CFL}_{1}$, the CFL number of the coarse levels is determined by $\mathrm{CFL}_{L}=\mathrm{CFL}_{1} \sqrt{(L)}$.

\section{Numerical Results}

To verify the preconditioned HLLE+ scheme for low Mach number flows, several well-known problems in both low and high Mach number flows were computed and compared with the previous results and experimental data. In this paper, Roe $(\mathrm{P})$ and HLLE + (P) denote the preconditioned Roe and HLLE + schemes, respectively. 
a)

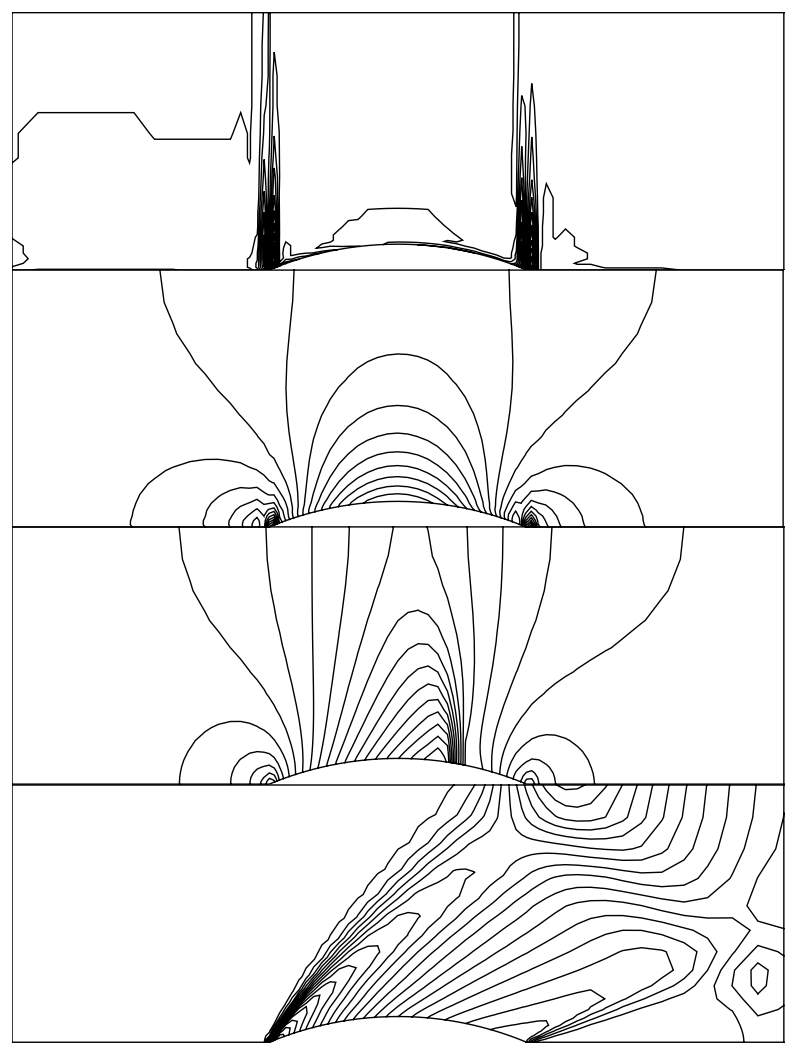

d)

Fig. 2 HLLE + pressure perturbation contours with Mach number a) 0.001 , b) 0.1 , c) 0.675 , d) 2.0 .

\section{Inviscid Flow Past a Bump}

To examine the effect of preconditioning, we computed inviscid flows past a $10 \%$ arc bump at various Mach numbers. The coarse grid used consists of 65 points in the flow direction and 17 points in the normal direction. For the inflow and outflow boundary conditions, applied is a simplified set of noncharacteristic conditions, which yields almost the same results as the characteristic boundary conditions [23].

Figure 2 shows the pressure perturbation contours of the HLLE+ scheme without preconditioning, whereas Fig. 3 shows the same data for the HLLE+ scheme with preconditioning. As shown in Fig. 2, the HLLE+ scheme as well as the Roe scheme gives a contaminated solution at $M=0.001$ and 0.1 , where no visible differences are found at $M=0.675$ and 2.0. This unphysical behavior of common shock-capturing schemes can be eliminated by implementing a low Mach number preconditioning method. The pressure distributions in Figs. $3 \mathrm{a}$ and $3 \mathrm{~b}$ show nearly symmetric solutions of the HLLE + (P) scheme. The preconditioning method can effectively produce a reasonable result up to $M=10^{-5}$ (not shown).

The effect of the preconditioning is obviously found in the convergence histories shown in Figs. 4 and 5. A four-level multigrid method with a CFL of 3 is applied to all inviscid bump computations. The failed or very slow convergence in Fig. 4 is common in shockcapturing schemes when preconditioning is not applied. For the low Mach number cases in Fig. 5, the convergence of the HLLE + (P) scheme is nearly independent of Mach numbers. In contrast, the order of the convergence varies with the freestream Mach number.

Figure 6 compares the pressure coefficient distribution of each spatial scheme. The Roe $(\mathrm{P})$ and the HLLE $+(\mathrm{P})$ schemes produce almost the same results. The Roe and the HLLE+ schemes generate unphysical solutions at $M=0.1$ that are kinked at the leading and trailing edges of the bump. Although the results for the transonic and supersonic cases are not shown, the results from the preconditioned schemes are nearly the same as the nonpreconditioned ones. a)

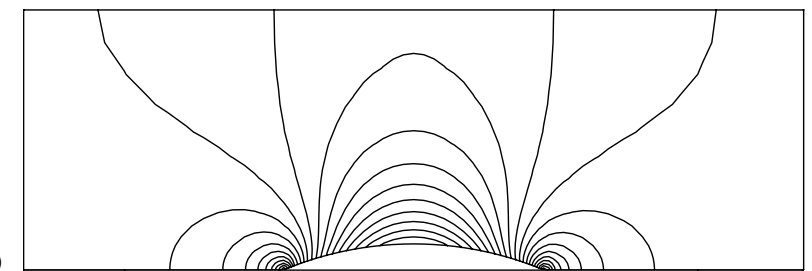

b)

c)

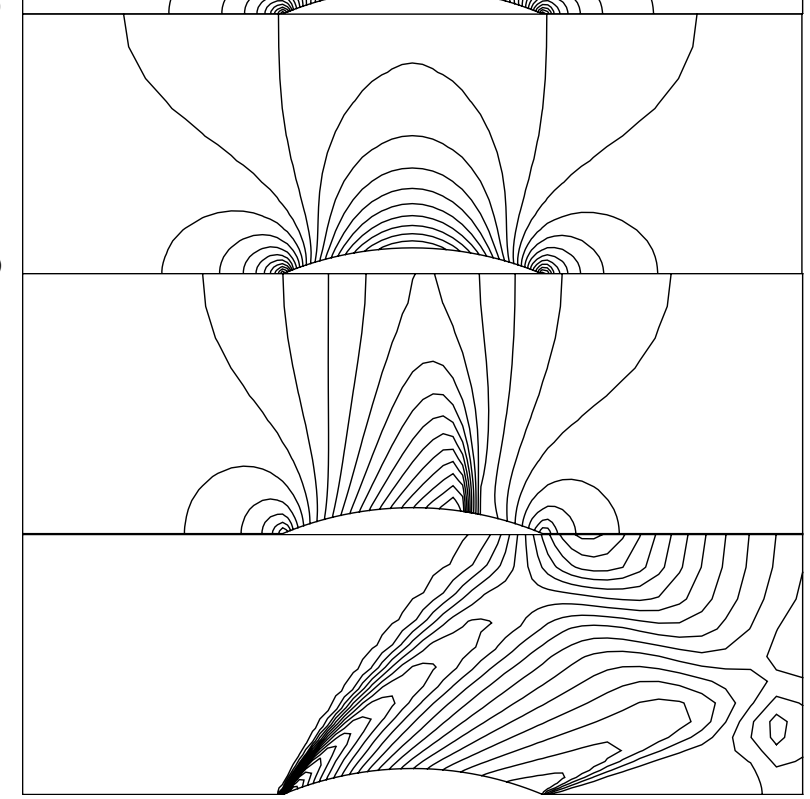

d)

Fig. 3 HLLE + (P) pressure perturbation contours with Mach number a) 0.001, b) 0.1 , c) 0.675 , d) 2.0 .

\section{Supersonic Blunt-Body Flow}

The next test problem concerns a 2-D supersonic inviscid flow past a cylinder. This kind of test is used to verify the accuracy of any spatial scheme for supersonic viscous flows [6]. A $65 \times 65$ grid is used and a freestream Mach number is 15.0. Here, the limiter at the MUSCL interpolation is not van-Albada but minmod having discrete manner of derivative in certain regions [18]. Figure 7a shows the well-known shock instability, namely carbuncle. This instability has forced Godunov-type schemes to use an entropy correction or a switching mechanism [5,6]. However, the Roe (P) and HLLE + (P)

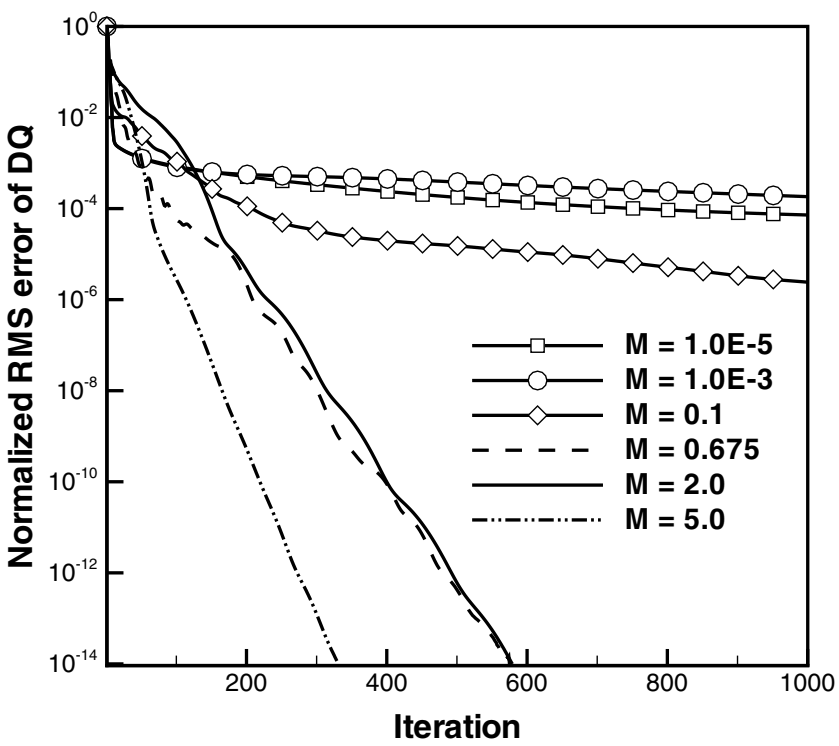

Fig. 4 Convergence history for the inviscid bump with Mach number: HLLE+. 


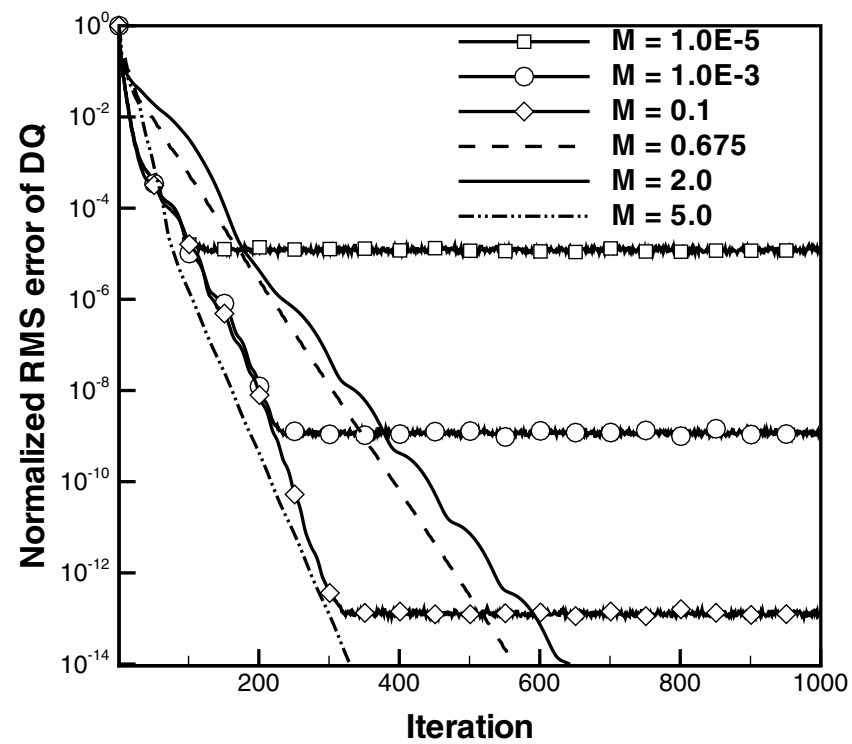

Fig. 5 Convergence history for the inviscid bump with Mach number: HLLE + (P).

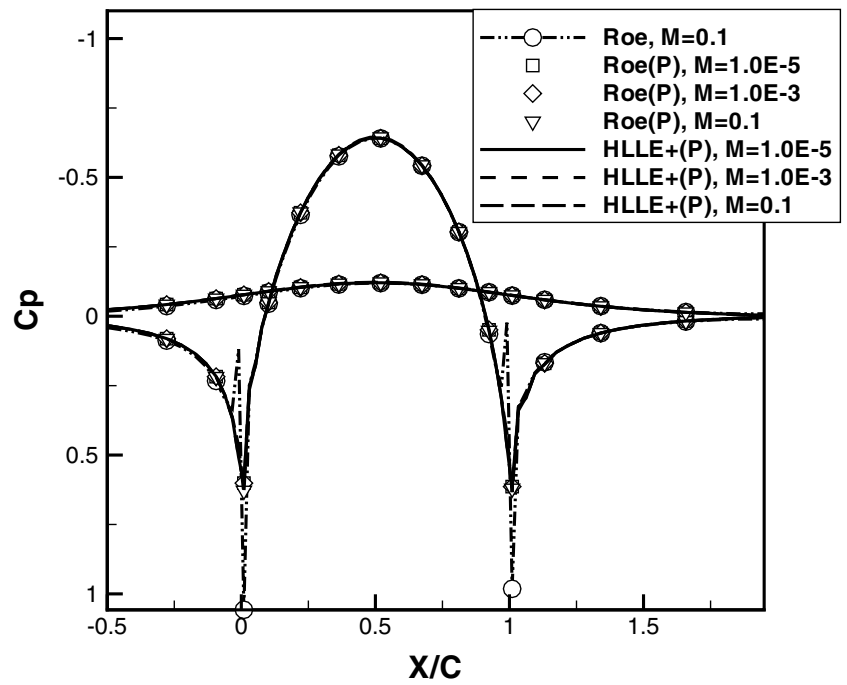

Fig. 6 Comparison of pressure coefficient distributions on the bump surface.

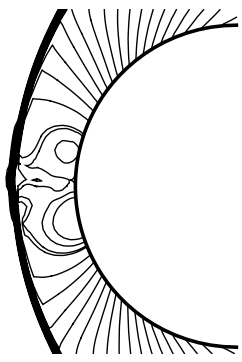

a) $\mathbf{R o e}$

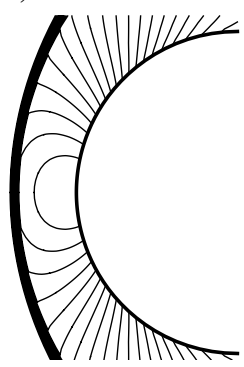

c) $\operatorname{Roe}(\mathbf{P})$

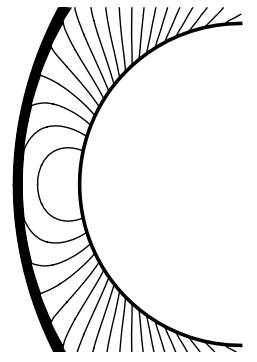

b) HLLE+

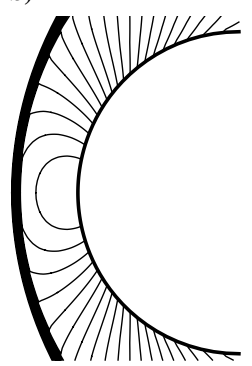

d) HLLE+(P)
Fig. 7 Comparison of pressure contours with and without preconditioning.

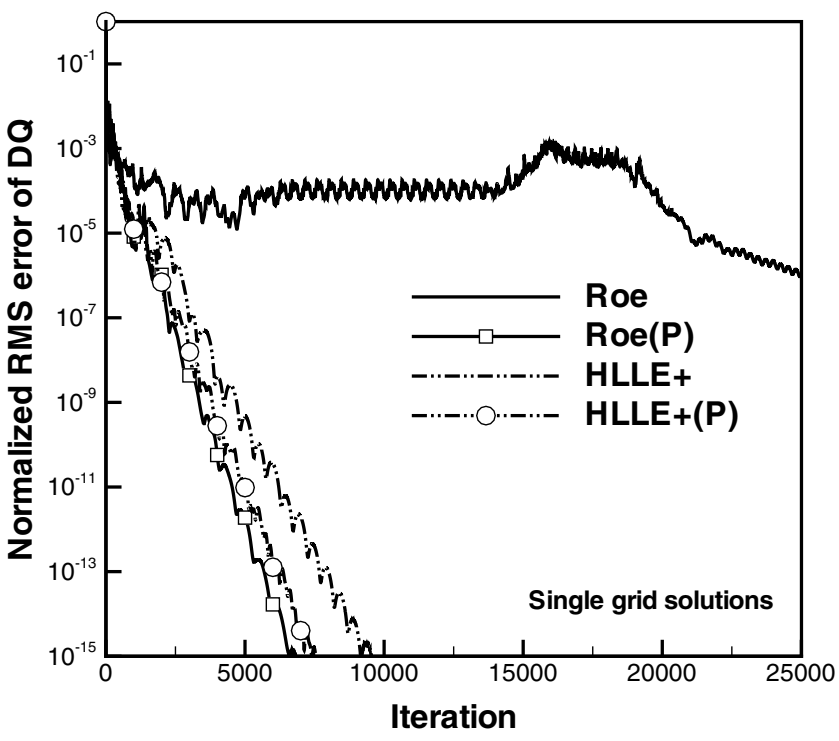

Fig. 8 Convergence history of blunt-body flow.

schemes do not suffer from this instability. It is noted that the HLLE + (P) scheme has no switching mechanism. In the case of the Roe $(\mathrm{P})$ method, the minmod limiter contributes to the elimination of carbuncle rather than the preconditioning itself. It can be confirmed that similar results are obtained by employing a part of Liou's theory [5] without the minmod limiter. That is, the coefficient of the pressure at the numerical dissipation should be zero at the supersonic regimes.

The most important point of the present application is to confirm effects of the proposed definition of the reference velocity $U_{r}$. $\sqrt{\left|p^{\prime}\right| / \rho}$ term in the Eq. (8) makes the preconditioning solver calculate robustly under supersonic flow or multigrid condition. As mentioned before, $\boldsymbol{U}_{\text {cut }}$ of the modified reference velocity enables the preconditioning effect to be activated in the vicinity of locally subsonic regions in globally supersonic flows. If $\boldsymbol{U}_{\text {cut }}$ uses $\boldsymbol{U}_{\infty}$ only, original global cutoff definition overcomes the local speed of sound even at subsonic domains when the freestream Mach number is 15 . However, once $a_{\infty}$ is accompanied with $\boldsymbol{U}_{\infty}$ as the proposed global cutoff definition, the $K \boldsymbol{U}_{\text {cut }}$ term has smaller magnitude than other terms including the local speed of sound. From the present numerical experiment, $K \boldsymbol{U}_{\text {cut }}$ [Eq. (8)] $<|\boldsymbol{U}|<\sqrt{\left|p^{\prime}\right| / \rho}<a$ behind shock. In addition to these advantages, the modified limitations not only have harmless solutions for other subsonic implementations, the convergence rates of the preconditioned schemes in Fig. 8 are also much better than those of the original schemes.

Consequently, preconditioning formulations are more effective than nonpreconditioning forms even if a Liou's theory is a dominant solution for the shock instability in a point view of carbuncle only. This phenomenon gives an interesting insight into the relation between the supersonic shock instability and the low Mach number asymptotic. The numerical consequences, however, should be verified by an asymptotic analysis such as Guillard and Viozat's analysis [16].

\section{Steady Flow in a Lid-Driven Cavity}

As an example of the low Mach number viscous flows, a flow in a lid-driven cavity is computed. The Mach number of the moving lid is 0.001 . Three Reynolds numbers of 1, 100, and 1000 are considered and the no-slip isothermal wall boundary condition is applied with a zero pressure gradient. Figure 9 displays the streamline contours for the three Reynolds numbers. The streamline patterns show a large primary vortex coupled with two secondary vortices at the bottom corners. To verify the accuracy of the solutions, the $U$ - and $V$ velocities at the centerlines of the cavity are displayed in Figs. 10 and 11. The results obtained from different grids agree well with the benchmark solutions of Ghia et al. [24]. Figures 12 and 13 show the convergence histories of the root-mean-square error of $\Delta Q$, which is 


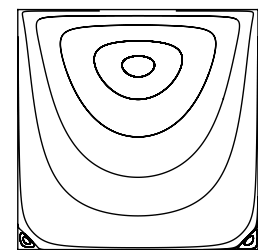

$\mathrm{Re}=1$

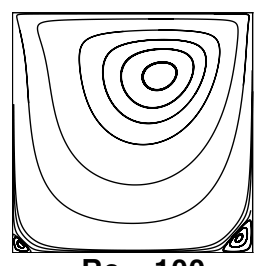

$\operatorname{Re}=100$

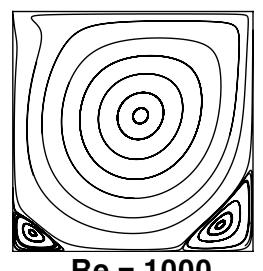

$\mathrm{Re}=1000$
Fig. 9 Streamline contours for flow in a lid-driven cavity.

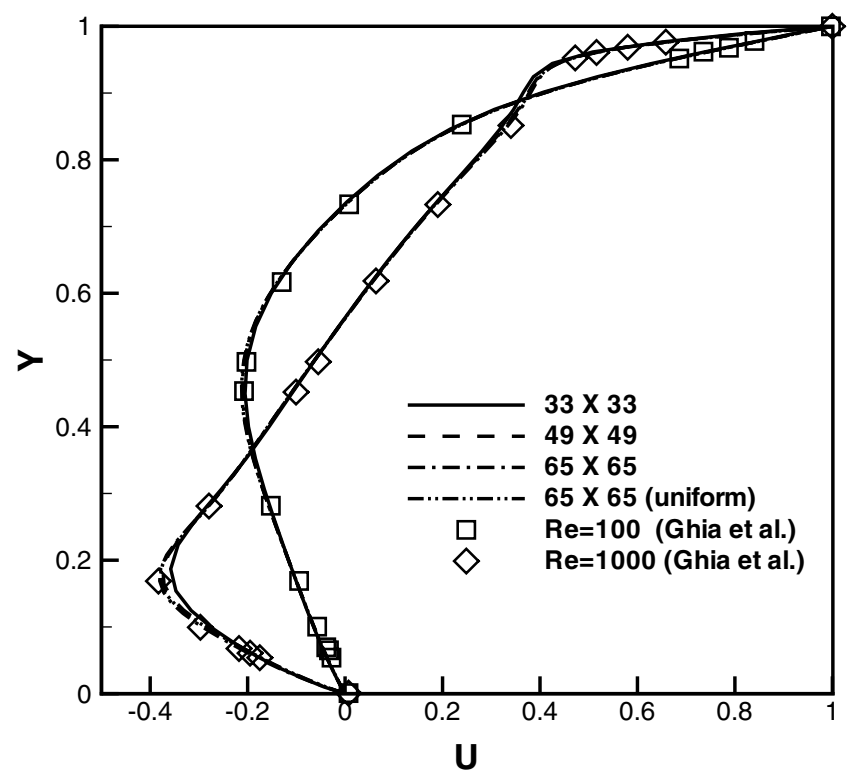

Fig. $10 U$-velocity along vertical centerline for lid-driven cavity flow.

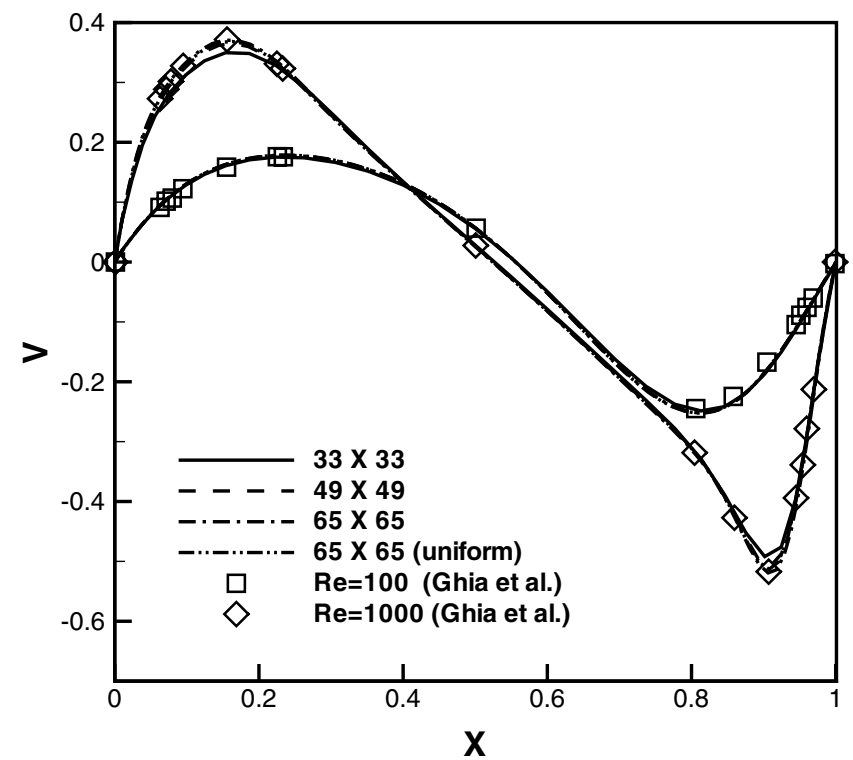

Fig. $11 V$-velocity along horizontal centerline for lid-driven cavity flow.

normalized by the initial $\Delta Q_{0}$ when four-level multigrid with a CFL of 5 is applied. Except in a few cases, the convergence rate does not depend on the Reynolds number, the grid size, or the aspect ratio. The convergence rate largely depends on the definition of the reference velocity, especially for low Mach number viscous flows. Although special treatments for viscous preconditioning have been devised over the last decade [9], they have not yet been implemented.

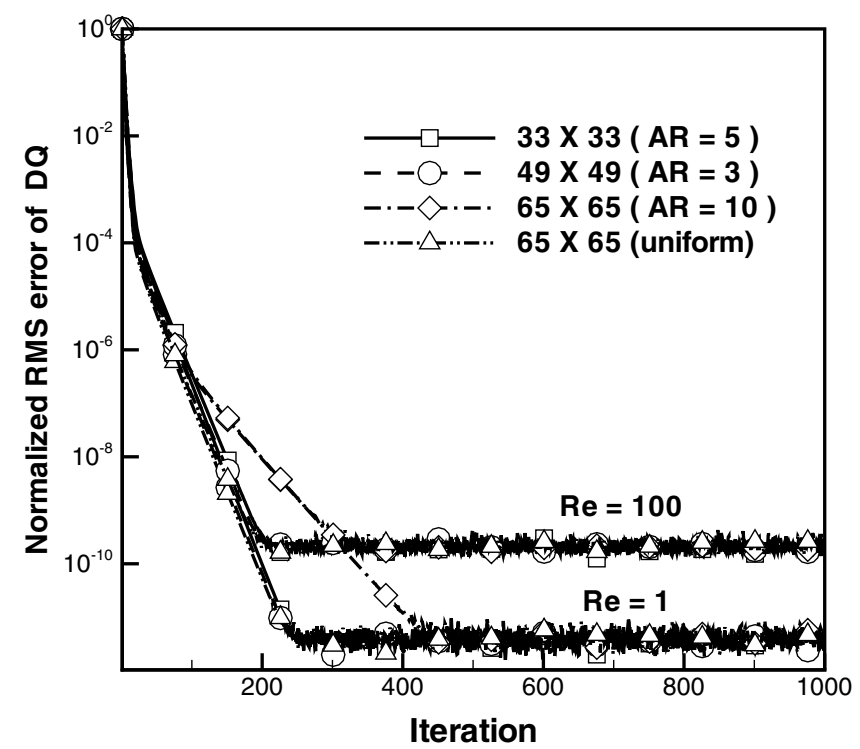

Fig. 12 Convergence history for driven cavity for $R e=1$ and 100 with grid size.

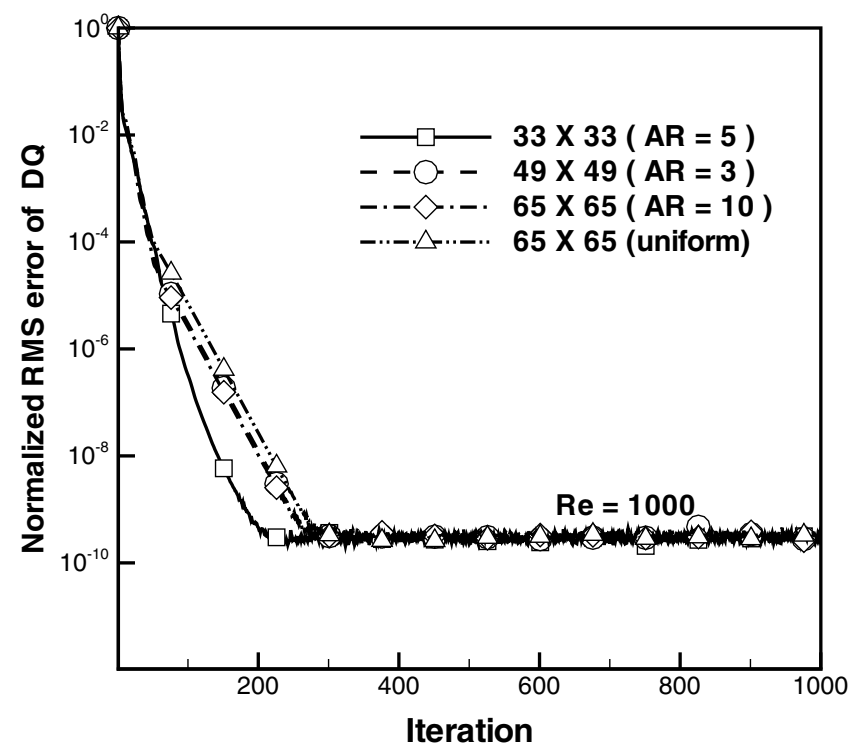

Fig. 13 Convergence history for driven cavity for $R e=1000$ with grid size.
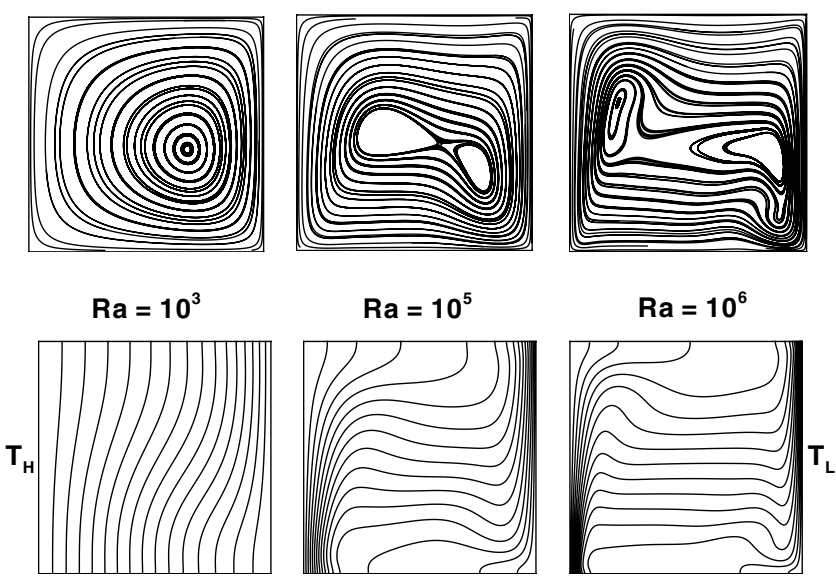

Fig. 14 Streamline and isotemperature contours in thermally driven cavity for different Rayleigh numbers. 
Table 1 Nusselt numbers on the hot wall extrapolated with Richardson's method with grid size

\begin{tabular}{lccc}
\hline \hline$N$ & $\overline{N u}, R a=10^{3}$ & $\overline{N u}, R a=10^{5}$ & $\overline{N u}, R a=10^{6}$ \\
\hline 65 & 1.0974143 & 4.4675517 & 8.7412005 \\
129 & 1.0936196 & 4.4278758 & 8.5951033 \\
257 & 1.0906887 & 4.4140846 & 8.5586672 \\
extrap. & 1.0807433 & 4.4067367 & 8.5465609 \\
$\alpha$ & -0.3726170 & -1.5245111 & -2.0034899 \\
$C$ & 0.0012579 & $1.5566613 \mathrm{E}-06$ & $1.7977670 \mathrm{E}-07$ \\
\hline
\end{tabular}

\section{Steady Flow in a Thermally Driven Cavity}

The next test problem is a buoyancy-driven flow in a square cavity. The configuration comprised two vertical walls at temperature $T_{H}$ and $T_{L}$ along with two adiabatic horizontal walls. The solution to this complex problem depends on a variety of flow parameters, such as the Rayleigh number $\left[R a=\rho^{2} g \beta\left(T_{H}-T_{L}\right) L^{3} C_{p} /(\mu k)\right]$, the aspect ratio of the cavity, and a temperature difference parameter $\left[\epsilon=\left(T_{H}-T_{L}\right) /\left(T_{H}+T_{L}\right)\right]$. Here $\beta$ is the thermal expansion coefficient, $g$ is the magnitude of the gravitational field, $L$ is the length of the cavity walls, and $\mu$ and $k$ are the dynamic viscosity and thermal conductivity, respectively.

For the present study, three Rayleigh numbers, $R a=10^{3}, 10^{5}$, and $10^{6}$, are considered with a temperature difference parameter $\epsilon=0.6$. The grid sizes are $65 \times 65,129 \times 129$, and $257 \times 257$. The maximum aspect ratios (AR) of each grid are 10, 40, and 120 . Figure 14 displays streamlines and temperature contours for each Rayleigh number. The center of the vortex at $R a=10^{3}$ shifted toward the lower and cold wall. A recirculating roll is driven by the generation of vorticity because of the horizontal temperature gradient. At $R a=10^{5}$ and $10^{6}$ the secondary vortices are generated because of the adverse temperature gradient [9].

For each Rayleigh number case, a grid refinement study is performed in line with the approach of Vierendeels et al. [15]. The results are summarized in Table 1 . The extrapolated values are computed with Richardson's extrapolation method:

$$
\begin{gathered}
f_{\text {extrap }}=f_{h}-C h^{\alpha} \\
\alpha=\frac{\ln \left(f_{h}-f_{h / 2}\right) /\left(f_{h / 2}-f_{h / 4}\right)}{\ln (2)}
\end{gathered}
$$

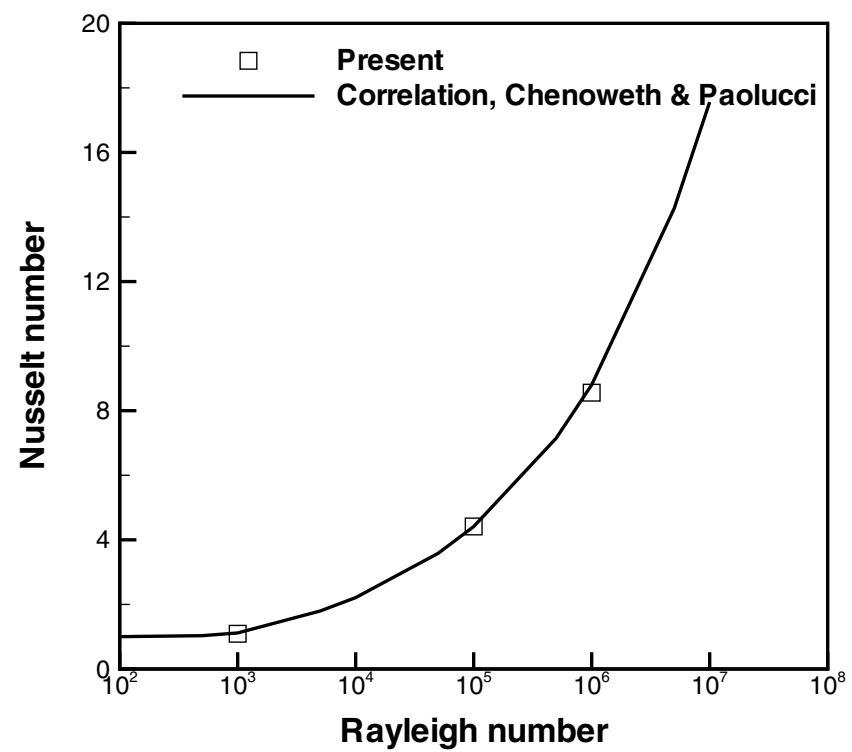

Fig. 15 Comparison of the extrapolated Nusselt number with a correlation.

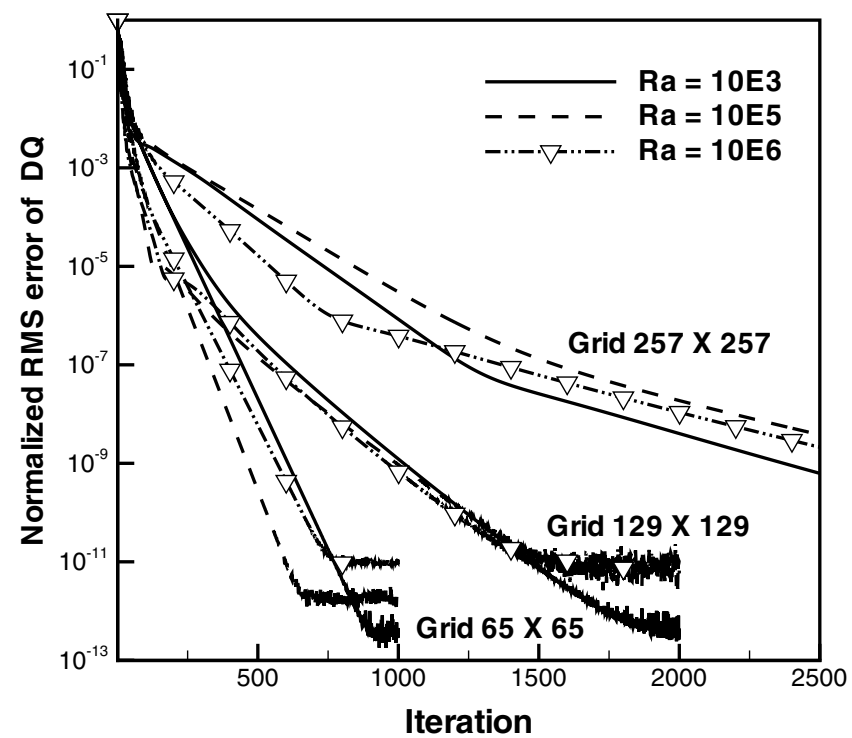

Fig. 16 Convergence history for thermally driven cavity with grid size.

$$
C=\frac{f_{h}-f_{h / 2}}{h^{\alpha}\left(1-2^{-\alpha}\right)}
$$

where $h$ is given by $1 / N$ and, $N$ is the number of grid cells in a single direction. We used $h=1 / 257$. The computed $\alpha$ values show the quadratic grid convergence. As shown in Fig. 15, the extrapolated Nusselt values are in an excellent agreement with the correlation given by Chenoweth and Paolucci [25].

The convergence histories for each grid size are displayed in Fig. 16. Although the convergence rate does not depend largely on the Rayleigh number for each grid, the rate slows as the grid aspect ratio increases. The implicit multigrid method mainly causes this dependence but a special viscous preconditioning [9] can alleviate the convergence degradation.

\section{Conclusions}

We extended the HLLE + scheme in conjunction with timederivative preconditioning for low Mach number viscous flows. Limitations on the reference velocity must be carefully imposed onto a preconditioned scheme, especially when it is developed for flows at all Mach numbers. Proposed additional limitations using pressure perturbation and modified global cutoff terms are shown to lead to an improvement of the robustness of the preconditioning system. The inviscid bump and cylinder problems were tested to assess the performance of our scheme. Parametric studies for viscous cavity problems reveal that the present multigrid algorithm gives a good convergence characteristic that is essentially independent of Reynolds numbers and Rayleigh numbers.

\section{Appendix: Preconditioned Matrices}

Following formulations $[10,16,22]$ include components of the antidiffusive term for the original and preconditioned HLLE+ schemes.

\section{HLLE + method}

An inviscid Jacobian $\boldsymbol{A}$ and a conserved variable set $\boldsymbol{W}$ can be defined as follows when the governing equation is two-dimensional Euler equations. 


$$
\begin{aligned}
\boldsymbol{W}=\left[\begin{array}{llll}
\rho & \rho u & \rho v & \rho e
\end{array}\right]^{T} \quad \boldsymbol{A} & =\left[\begin{array}{cccc}
0 & \kappa_{x} & \kappa_{y} & 0 \\
\kappa_{x}(\gamma-1) \phi-u \boldsymbol{U} & \boldsymbol{U}-(\gamma-2) \kappa_{x} u & \kappa_{y} u-(\gamma-1) \kappa_{x} v & (\gamma-1) \kappa_{x} \\
\kappa_{y}(\gamma-1) \phi-v \boldsymbol{U} & \kappa_{x} v-(\gamma-1) \kappa_{y} u & \boldsymbol{U}-(\gamma-2) \kappa_{y} v & (\gamma-1) \kappa_{y} \\
(\gamma-1) \phi \boldsymbol{U}-H \boldsymbol{U} & H \kappa_{x}-(\gamma-1) u \boldsymbol{U} & H \kappa_{y}-(\gamma-1) v \boldsymbol{U} & \gamma \boldsymbol{U}
\end{array}\right] \\
\phi & =\left(u^{2}+v^{2}\right) / 2, \quad \boldsymbol{U}=u \kappa_{x}+v \kappa_{y}, \quad \kappa=\xi, \eta
\end{aligned}
$$

A Jacobian matrix $\boldsymbol{A}$ is related with the eigensystem constituted with the right eigenvectors and eigenvalues.

$$
\boldsymbol{A}=\boldsymbol{T} \Lambda \boldsymbol{T}^{-1}
$$

where

$$
\begin{gathered}
\boldsymbol{\Lambda}(\boldsymbol{A})=D\left[\boldsymbol{U}, \boldsymbol{U}+a\left(\kappa_{x}^{2}+\kappa_{y}^{2}\right), \boldsymbol{U}-a\left(\kappa_{x}^{2}+\kappa_{y}^{2}\right), \boldsymbol{U}\right] \\
=\left[\begin{array}{cccc}
\boldsymbol{U} & 0 & 0 & 0 \\
0 & \boldsymbol{U}+a\left(\kappa_{x}^{2}+\kappa_{y}^{2}\right) & 0 & 0 \\
0 & 0 & \boldsymbol{U}-a\left(\kappa_{x}^{2}+\kappa_{y}^{2}\right) & 0 \\
0 & 0 & 0 & \boldsymbol{U}
\end{array}\right]
\end{gathered}
$$

Each element of the right eigenvectors $\boldsymbol{T}^{(p)}(p=1,2,3,4)$ and $\alpha^{(p)}$ the coefficients of the projection of $\Delta \boldsymbol{W}\left(=\boldsymbol{W}_{r}-\boldsymbol{W}_{l}\right)$ onto $\boldsymbol{T}^{(p)}$ in Eq. (11) can be written as follows as well:

$$
\begin{gathered}
\alpha^{(1)}(\Delta \boldsymbol{W})=\Delta \rho-\frac{\Delta p}{a^{2}}, \quad \alpha^{(2)}(\Delta \boldsymbol{W})=\frac{1}{2 a}\left(\frac{\Delta p}{a}+\rho \Delta \tilde{\boldsymbol{U}}\right) \\
\alpha^{(3)}(\Delta \boldsymbol{W})=\frac{1}{2 a}\left(\frac{\Delta p}{a}-\rho \Delta \tilde{\boldsymbol{U}}\right), \quad \alpha^{(4)}(\Delta \boldsymbol{W})=\rho \Delta \tilde{\boldsymbol{V}} \\
\boldsymbol{T}^{(1)}(\boldsymbol{W})=\left[\begin{array}{c}
1 \\
u \\
v \\
\phi
\end{array}\right], \quad \boldsymbol{T}^{(2)}(\boldsymbol{W})=\left[\begin{array}{c}
1 \\
u+a \tilde{\kappa}_{x} \\
v+a \tilde{\kappa}_{y} \\
H+a \tilde{\boldsymbol{U}}
\end{array}\right] \\
\boldsymbol{T}^{(3)}(\boldsymbol{W})=\left[\begin{array}{c}
u-a \tilde{\kappa}_{x} \\
v-a \tilde{\kappa}_{y} \\
H-a \tilde{\boldsymbol{U}}
\end{array}\right], \quad \boldsymbol{T}^{(4)}(\boldsymbol{W})=\left[\begin{array}{c}
-\tilde{\kappa}_{y} \\
\tilde{\kappa}_{x} \\
\tilde{\boldsymbol{V}}
\end{array}\right]
\end{gathered}
$$

Here,

$$
\tilde{\boldsymbol{U}}=u \tilde{\kappa}_{x}+v \tilde{\kappa}_{y}, \quad \tilde{\boldsymbol{V}}=-u \tilde{\kappa}_{y}+v \tilde{\kappa}_{x}, \quad \tilde{\kappa}_{x}=\kappa_{x} / \sqrt{\kappa_{x}+\kappa_{y}}
$$

\section{Preconditioned HLLE+ Method}

In the case of the preconditioned HLLE+ scheme, the preconditioning matrix $\Gamma$ directly affects the eigenstructure. When the preconditioned Jacobian matrix $\boldsymbol{A}_{\Gamma}$ is defined to be

$$
\boldsymbol{A}_{\Gamma}=\Gamma^{-1} \boldsymbol{A}=\left[\begin{array}{cccc}
\beta \boldsymbol{U} & \rho \gamma T \beta \kappa_{x} & \rho \gamma T \beta \kappa_{y} & 0 \\
\kappa_{x} / \rho & \boldsymbol{U} & 0 & 0 \\
\kappa_{y} / \rho & 0 & \boldsymbol{U} & 0 \\
\frac{\boldsymbol{U}(\gamma-1)(\beta-1)}{\rho \gamma} & (\gamma-1) T \beta \kappa_{x} & (\gamma-1) T \beta \kappa_{y} & \boldsymbol{U}
\end{array}\right]
$$

then the following relation is satisfied

$$
\boldsymbol{A}_{\Gamma}=\boldsymbol{T}_{\Gamma} \Lambda_{\Gamma} \boldsymbol{T}_{\Gamma}^{-1}
$$

and eigenvalues and eigenvectors can be written as follows:

$$
\begin{aligned}
& \boldsymbol{\Lambda}_{\Gamma}\left(\boldsymbol{A}_{\Gamma}\right)=D\left[\boldsymbol{U}, u^{\prime}+a^{\prime}, u^{\prime}-a^{\prime}, \boldsymbol{U}\right]=D\left[\boldsymbol{U}, \frac{(1-\beta) \boldsymbol{U}}{2}\right. \\
& \left. \pm \sqrt{(1-\beta)^{2} \boldsymbol{U}^{2} / 2+U_{r}^{2}\left(\kappa_{x}^{2}+\kappa_{y}^{2}\right)}, \boldsymbol{U}\right] \\
& \boldsymbol{T}_{\Gamma}=\left[\begin{array}{cccc}
0 & \frac{\tilde{\boldsymbol{U}} \beta-\tilde{\Lambda}_{\Gamma}^{(3)}}{2 \tilde{\tilde{e}}^{\prime}} & \frac{\tilde{\Lambda}_{\Gamma}^{(2)}-\tilde{\boldsymbol{U}} \beta}{2 \tilde{a}^{\prime}} & 0 \\
0 & \frac{\tilde{\kappa}_{x}}{2 \rho \tilde{a}^{\prime}} & -\frac{\tilde{\boldsymbol{K}}_{x}}{2 \rho \tilde{a}^{\prime}} & -\tilde{\kappa}_{y} \\
0 & \frac{\tilde{\kappa}_{y}}{2 \rho \tilde{\sigma}^{\prime}} & -\frac{\tilde{\boldsymbol{K}}_{y}}{2 \rho \tilde{a}^{\prime}} & \tilde{\kappa}_{x} \\
1 & \frac{(\gamma-1) \tilde{\boldsymbol{U}} \beta-\tilde{\Lambda}_{\Gamma}^{(3)}}{2 \rho \gamma \tilde{a}^{\prime}} & \frac{(\gamma-1) \tilde{\Lambda}_{\Gamma}^{(2)}-\tilde{\boldsymbol{U}} \beta}{2 \rho \gamma \tilde{a}^{\prime}} & 0
\end{array}\right] \\
& \tilde{\Lambda}_{\Gamma}^{(2)}=\tilde{u}^{\prime}+\tilde{a}^{\prime}, \quad \tilde{\Lambda}_{\Gamma}^{(3)}=\tilde{u}^{\prime}-\tilde{a}^{\prime}
\end{aligned}
$$

$\alpha_{\Gamma}^{(p)}$ and $\boldsymbol{T}_{\Gamma}^{(p)}$ can be also induced such as the original HLLE+ scheme.

$$
\begin{gathered}
\alpha_{\Gamma}^{(1)}(\Delta \boldsymbol{W})=-\frac{T}{\rho}\left(\Delta \rho-\frac{\Delta p}{U_{r}^{2}}\right), \quad \alpha_{\Gamma}^{(2)}(\Delta \boldsymbol{W})=r\left(\frac{\Delta p}{r}+\rho \Delta \tilde{\boldsymbol{U}}\right) \\
\alpha_{\Gamma}^{(3)}(\Delta \boldsymbol{W})=s\left(\frac{\Delta p}{s}-\rho \Delta \tilde{\boldsymbol{U}}\right), \quad \alpha^{(4)}(\Delta \boldsymbol{W})=\Delta \tilde{\boldsymbol{V}} \\
\Gamma \boldsymbol{T}_{\Gamma}^{(1)}(\boldsymbol{W})=-\frac{\rho}{T}\left[\begin{array}{c}
u \\
v \\
\phi
\end{array}\right], \quad \Gamma \boldsymbol{T}_{\Gamma}^{(2)}(\boldsymbol{W})=\frac{1}{r 2 \tilde{a}^{\prime}}\left[\begin{array}{c}
1 \\
u+r \tilde{\kappa}_{x} \\
v+r \tilde{\kappa}_{y} \\
H+r \tilde{\boldsymbol{U}}
\end{array}\right] \\
\Gamma \boldsymbol{T}_{\Gamma}^{(3)}(\boldsymbol{W})=\frac{1}{s 2 \tilde{a}^{\prime}}\left[\begin{array}{c}
u-s \tilde{\kappa}_{x} \\
v-s \tilde{\kappa}_{y} \\
H-s \tilde{\boldsymbol{U}}
\end{array}\right], \quad \Gamma \boldsymbol{T}_{\Gamma}^{(4)}(\boldsymbol{W})=\rho\left[\begin{array}{c}
0 \\
-\tilde{\kappa}_{y} \\
\tilde{\kappa}_{x} \\
\tilde{\boldsymbol{V}}
\end{array}\right]
\end{gathered}
$$

where

$$
r=\frac{U_{r}^{2}}{\tilde{\boldsymbol{U}} \beta-\tilde{\Lambda}_{\Gamma}^{(3)}}, \quad s=\frac{U_{r}^{2}}{\tilde{\Lambda}_{\Gamma}^{(2)}-\tilde{\boldsymbol{U}} \beta}
$$

\section{References}

[1] Hartern, A., Lax, P. D., and Van Leer, B., "On Upstream Differencing and Godunov-Type Schemes for Hyperbolic Conservation Laws," SIAM Review, Vol. 25, No. 1, 1983, pp. 35-61.

[2] Einfeldt, B., "On Godunov-Type Methods for Gas Dynamics," SIAM Journal on Numerical Analysis, Vol. 25, No. 2, 1988, pp. 294-318.

[3] Einfeldt, B., Munz, C. D., Roe, P. L., and Sjögreen, B., "On GodunovType Methods Near Low Densities," Journal of Computational Physics, Vol. 92, No. 2, 1991, pp. 273-295.

[4] Roe, P. L., "Characteristic Based Schemes for the Euler Equation," Annual Review of Fluid Mechanics, Vol. 18, 1986, pp. 337-365.

[5] Liou, M.-S., "Mass Flux Schemes and Connection to Shock Instability," Journal of Computational Physics, Vol. 160, No. 2, 2000, pp. 623-648.

[6] Park, S. H., and Kwon, J. H., "On the Dissipation Mechanism of Godunov-Type Schemes," Journal of Computational Physics, Vol. 188, No. 2, 2003, pp. 524-542.

[7] Viviand, H., "Pseudo-Unsteady Systems for Steady Inviscid Flow Calculations," Numerical Methods for the Euler Equations of Fluid 
Dynamics, edited by F. Angrand, A. Dervieux, J. A. Desideri, and R. Glowinski, SIAM, Philadelphia, 1985, p. 334.

[8] Van Leer, B., Lee, W. T., and Roe. P. L., "Characteristic Time-Stepping or Local Preconditioning of the Euler Equations," AIAA Paper 911552, June 1991

[9] Choi, Y.-H., and Merkle, C. L., "The Application of Preconditioning in Viscous Flows," Journal of Computational Physics, Vol. 105, No. 2, 1993, pp. 207-223.

[10] Weiss, J. M., and Smith, W. A., "Preconditioning Applied to Variable and Constant Density Flows," AIAA Journal, Vol. 33, No. 11, 1995, pp. 2050-2057.

[11] Turkel, E., "Preconditioning Techniques in Computational Fluid Dynamics," Annual Review of Fluid Mechanics, Vol. 31, 1999, pp. $385-416$

[12] Weiss, J. M., Maruszewski, J. P., and Smith, W. A., "Implicit Solution of Preconditioned Navier-Stokes Equations Using Algebraic Multigrid," AIAA Journal, Vol. 37, No. 1, 1999, pp. 29-36.

[13] Luo, H., Baum, J. D., and Lohner, R., "Extension of Harten-Lax-van Leer Scheme for Flows at All Speeds," AIAA Journal, Vol. 43, No. 6, 2005, pp. 1160-1166.

[14] Edwards, J. R., and Liou, M.-S., "Low-Diffusion Flux-Splitting Methods for Flows at All Speeds," AIAA Journal, Vol. 36, No. 9, 1998, pp. 1610-1617.

[15] Vierendeels, J., Merci, B., and Dick, E. "Blended AUSM+ Method for All Speeds and All Grid Aspect Ratios," AIAA Journal, Vol. 39, No. 12, 2001, pp. 2278-2282.

[16] Guillard, H., and Viozat, C., "On the Behaviour of Upwind Schemes in the Low Mach Number Limit," Computers \& Fluids, Vol. 28, No. 1, 1999, pp. 63-86.

[17] Venkateswaran, S., Li, D., and Merkle, C. L., "Influence of Stagnation
Regions on Preconditioned Solutions at Low Speeds," AIAA Paper 2003-0435, Jan. 2003.

[18] Anderson, W. K., Tomas, J. L., and Van Leer, B., "Comparison of Finite Volume Flux Vector Splittings for the Euler Equations," AIAA Journal, Vol. 24, No. 9, 1986, pp. 1453-1460.

[19] Pulliam, T. H., and Chaussee, D. S., "A Diagonal Form of an Implicit Approximate-Factorization Algorithm," Journal of Computational Physics, Vol. 39, No. 2, 1981, pp. 347-363.

[20] Ni, R.-H., "A Multiple-Grid Scheme for Solving the Euler equations," AIAA Journal, Vol. 20, No. 11, 1982, pp. 1565-1571.

[21] Dailey, L. D., and Pletcher, R. H., "Evaluation of Multigrid Acceleration for Preconditioned Time-Accurate Navier-Stokes Algorithms," Computers \& Fluids, Vol. 25, No. 8, 1996, pp. 791-811.

[22] Jespersen, D., Pulliam, T., and Buning, P., "Recent Enhancements to OVERFLOW," AIAA Paper 97-0644, Jan. 1997.

[23] Turkel, E., Radespiel, R., and Kroll, N., "Assessment of Preconditioning Methods for Multidimensional Aerodynamics," Computers \& Fluids, Vol. 26, No. 6, 1997, pp. 613-634.

[24] Ghia, U., Ghia, K. N., and Shin, C. T., "High-Re Solutions for Incompressible Flow Using the Navier-Stokes Equations and a Multigrid Method," Journal of Computational Physics, Vol. 48, No. 3, 1982, pp. 387-411.

[25] Chenoweth, D. R., and Paolucci, S., "Natural Convection in an Enclosed Vertical Air Layer with Large Horizontal Temperature Differences," Journal of Fluid Mechanics, Vol. 169, Aug. 1986, pp. 173-210.

G. Candler

Associate Editor 